\title{
RECENT ADVANCES IN OUR KNOWLEDGE OF MEASLES
}

\author{
JOHN F. ANDERSON \\ Director Hygienic Laboratory \\ AND \\ JOSEPH GOLDBERGER
}

Passed Assistant Surgeon, U. S. Public Health and Marine-Hospital Service WASHINGTON, D. C.

While it has been quite the general belief for years that the infection of measles is contained in the blood and in the nasal and buccal secretions and, perhaps, in the "scales," it is surprising on what inconclusive data this belief has been based.

Previous to 1905 , the data as to the infectivity of the blood, while highly suggestive, were by no means conclusive; but in that year Hektoen reported the infection of two men with measles by inoculation with an ascitic broth culture of blood drawn from human cases of the disease during the first thirty hours of the eruption. These results left no doubt as to the presence of the virus of measles in the blood during at least the first thirty hours of the eruptive period.

The state of our knowledge as to the presence of the virus in the nasal and buccal secretions was even more unsatisfactory than that in regard to the blood, although clinical observations and Mayr's experiments on children had made it fairly certain that these secretions were infective for man, and the same author's negative experiments with the "scales" had also shown that it was fairly certain that these did not contain the virus of the disease.

The literature of measles contains many reports of attempts to produce measles by various procedures in animals other than monkeys, but Josins ${ }^{1}$ seems to have been the first to attempt to inoculate the monkey with measles. His work had been inspired by Chavigny's ${ }^{2}$ reported observation of a monkey that had developed measles after having been in close contact with a case of the disease in the person of its keeper. In 1898 Josias reported attempts to infect monkeys with measles by various methods and he apparently obtained positive results in some instances.

1. Josias, Albert: Recherches expérimentale sur la transmissibilité de la rougeole aux animaux. Med. moderne, Paris, 1898, ix, p. 153.

Mayr, Frantz: Beobachtungen über Masern, etc. Zeitschr. d. k. k. Gesellsch. d. Aerzte zu Wien, 1852, i, 13; Masern. Virchow's Handb. der special Path. u. Therap., 1860, iii, Part 3, p. 106.

2. Chavigny, Paul: Un cas de rougeole chez un singe. Bull. méd. Paris, 1898, xii, p. 334 . 
Grunbaum ${ }^{3}$ in 1904 reported attempts to infect two chimpanzees by various means, including the injection of blood drawn from the median basilic vein of patients with measles, but he regarded his results as negative.

On account of the prominent place occupied by measles in our morbidity and mortality statistics, it appeared to us highly important that studies be made on the disease to determine its cause, means of transmission and prevention. In view of the apparent insusceptibility of the lower animals to measles it seemed to us most desirable that the susceptibility of the monkey to the disease be thoroughly tried out.

\section{AUTHORS' HXPERINEST.L WORK}

In pursuance of this plan we began our work ${ }^{4}$ on experimental measles early in the summer of 1910 by inoculating two rhesus monkeys with blood from a patient with measles. In each of the animals a slight rise in temperature, eleven days after inoculation, was noted, but an interpretation of its significance was not possible.

With blood from our second patient we also inoculated two monkeys with a result similar to our first case. With blood from our third patient we inoculated three monkeys. Ten days after inoculation there was noted a slight rise in the temperature of one of the animals. At the same time there appeared a few papules on the face, brows and chin with a diffuse erythema of the brows and eyelids. 'The rise in temperature was not sustained. At the end of four days the eruption had perceptibly faded, and a fine branny scaling was noted at the site of the fading papules. This result we were inclined to consider as indicating a mild attack of measles, even though an attempt at passage with blood drawn soon after the eruption was first noted, resulted negatively.

The thought was constantly present in our minds from the beginning of our work with measles that we should obtain blood for inoculation as early in the disease as possible, and fortunately we saw our fourth patient at not less than fourteen hours after the eruption first appeared. With blood drawn from this patient about fourteen hours after the eruption was

3. Grumbaum, A. S.: Some experiments on enterica, scarlet fever, and measles in the chimpanzee; a preliminary communication. Brit. Med. Jour., April 9, 1904, p. 817 .

4. Anderson, John F., and Goldberger, Joseph: Experimental Measles in the Monkey: A Preliminary Note. Pub. Health Rep., June 9, 1911, p. 847; Experimental Measles in the Monkey: A Supplemental Note. Pub. Health Rep., June 16, 1911; The Period of Infectivity of the Blood in Measles. Jour. Am. Med. Assn.: Tuly 8, 1911, p. 113; Goldberger, Joseph, and Anderson, John F.: An Experimental Demonstration of the Presence of the Virus of Measles in the Mixed Buccal and Nasal Secretions. Jour. Am. Med. Assn., August 5, 1911, p. 476; The Nature of the Virus of Measles. Jour. Am. Med. Assn., Sept. 16, 1911, p. 972; Anderson, John F., and Goldberger, Joseph: The Infectivity of the Secretions and the Desquamating Seales of Measles. Jour. Am. Med. Assn., Nov. 11, 1911, p. 1612. 
first ncted, and before the temperature had reached its maximum, we inoculated two rhesus monkeys. Ten days after inoculation the temperature of both animals showed a distinct rise and both developed an eruption. Blood drawn from the hearts of both of these monkeys was used for the inoculation of other monkeys, some of which developed febrile reactions and eruptions. This strain of measles virus was successfully propagated by blood inoculations through six monkey generations, when it was discontinued. The results obtained from the inoculations made from our fourth patient plainly showed us that the monkey was susceptible to measles by blood inoculations, but that this susceptibility was not acute and apparently was subject to quite considerable individual variation.

Since our work was reported three papers by different workers have appeared corroborating our results as to the susceptibility of the monkey to measles. Hektoen and Eggers, ${ }^{5}$ while mainly occupied in their work on experimental measles in the monkey with a study of the leukocytes, state that the general results of their experiments agree very well with those reported by Anderson and Goldberger.

Nicolle and Conseil ${ }^{6}$ reported the infection of the bonnet monkey with measles by the inoculation of blood drawn twenty-four hours before the first appearance of the eruption, and in a recent paper Lucas and Prizer also confirm our work as to the susceptibility of the monkey to measles by blood inoculation, and are the first to report the observation of Koplik spots in the monkey.

\section{LIMITATION OF INFECTIVITY}

The results obtained by these workers emphasize the statement made by us in our second paper ${ }^{4}$ as to the variation in susceptibility of monkeys to measles. but the variability in susceptibility of the animals to the disease while apparent early in our work, did not, in our opinion, fully explain the uncertain results obtained from the inoculations made from our first three patients. For that reason, after we had obtained such striking results from the inoculations made from the fourth patient, we carefully went over the notes of our first three cases to determine if possible whether this question of the susceptibility of the monkey was the only factor, or if there was not some other factor having something to do with the inconclusive results from our first three cases.

5. Hektoen, Ludvig, and Eggers, H. E.: Experimental Measles in the Monkey with Special Reference to the Leukocytes. Jour. Am. Med. Assn., Dec. 2, 1911, p. 1833.

6. Nicolle, Ch., and Conseil, E.: Reproduction expérimentale de la rougeole chez le bonnet chinois. Virulence du sang des malades 24 heures avant le début de la éruption. C. R. de Acad. Sci., Dec. 26, 1911, cliii. 
We found that the blood from our first three patients had been drawn after intervals of at least twenty-four to forty-two hours after the first appearance of the eruption, whereas the blood from our fourth patient had been drawn at the end of not less than fourteen hours after the eruption first appeared. The thought at once occurred to us that in measles we might have a disease in which the infectivity of the blood was more or less limited to the early period of the disease, as is the case, for example, in yellow fever, and that this limitation of infectivity of the blood was one of the factors concerned in the inconclusive outcome of our first three experiments.

In order to test this hypothesis, we drew blood from a patient with measles about six hours before the eruption first appeared. The patient was again bled exactly twenty-four hours later, or about eighteen hours after the first appearance of the eruption; the third bleeding was fortyfive hours after the second, and the fourth forty-eight hours after the third. At least two monkeys were inoculated from each bleeding as soon thereafter as possible. Both of the animals inoculated with blood drawn before the eruption appeared, gave a definite febrile reaction but no eruption; all three of the animals inoculated with the blood (one with serum, one with washed corpuscles, and one with defibrinated blood plus normal monkey blood) drawn eighteen hours after the first appearance of the eruption, gave well-marked reactions, including the presence of an eruption and involvement of the respiratory apparatus; no evidence of a reaction in one and at most a very doubtful reaction in the second of the two animals inoculated with blood drawn sixty-five hours after the eruption first began to appear; no indication of a reaction in either of the two monkeys inoculated with blood drawn 113 hours after the first appearance of the eruption.

These results taken with those reported by other observers since our work first appeared, point strongly to a period of infectivity of the blood beginning at least twenty-four hours before and continuing for about twenty-four hours after the first appearance of the exanthem. At the end of twenty-four hours from the first appearance of the eruption the infectivity of the blood for the rhesus monkey appears to be greatly lessened and becomes progressively less thereafter.

The existence of a more or less definitely limited period of infectivity of the blood in measles and the variation in susceptibility of the monkey to the disease would explain both the negative and positive results of Josias, would throw light on the inconclusive results of some of our earlier experiments and the reported failure of other workers, and make understandable the opinion almost universally held heretofore that the monkey is not susceptible to measles. 


\section{NATURE OF THE REACTION IN MONKEYS}

Before entering on a discussion of other results of our work with measles it would seem to be advisable to discuss briefly the nature of the reaction of the monkey to the disease. It has been shown that at least three species of the lower monkeys, Macacus rhesus, Macacus cynomolgus, and Macacus sinicus, are susceptible to measles, and it is quite probable that all monkeys are capable of taking the disease. Monkeys may be infected by introducing the virus into the body by any one of the usual methods of inoculation; but we have used, by preference, the intravenous method in most of our work. They also may contract the disease when placed in a cage with a sick monkey during, at least, the early period of the disease. When infection is produced by inoculation, the incubation period may be as short as five days or exceptionally as long as twenty-two days; but the average may be said to be approximately six to eight days.

The first indication of a reaction is usually a rise in temperature, especially a rise in the morning, with a sustained or higher reading in the afternoon; the temperature may remain at about the same level for four or five days and then drop rather sharply and remain down, unless there is a complicating bronchitis or pneumonia. The eruption usually begins to appear on about the third day after the rise in temperature begins, though it sometimes is coincident, or may be as late as the fourth or fifth day. In most cases it is first noticed on the chest or abdomen and may spread to the thighs, arms, and face. On account of the thick fur and dark skin the eruption is not so plainly discernible on the other portions of the body. It sometimes happens that the eruption begins to fade where it first appeared, while still appearing on other parts. As the eruption fades it may be followed by a fine branny desquamation. There is more or less variation, just as there is with human measles, in the appearance of the eruption; but the typical rash first appears as minute red spots which not infrequently coalesce, forming large patches; they soon begin to fade, but a stained spot may persist for several days. The eruption is not invariably present, but blood from monkeys with a distinct febrile reaction and with or without the respiratory symptoms has been found infectious. There is frequently coryza and coughing. In some instances the animal has been killed and found to have had a bronchopneumonia or even a lobar pneumonia. Some of our animals have presented as typical a picture of measles as will be seen in a well-marked case of the disease in human beings. Koplik spots have been observed by Lucas and Prizer. ${ }^{7}$

A monkey that has had a definite reaction is found to be immune to a subsequent inoculation with virulent blood.

7. Lucas, W. P., and Prizer, E. L.: An Experimental Study of Measles in Monkeys. Jour. Med. Research, Ipril, 1912. 


\section{NATURE OF THE VIRUS}

As before stated, the susceptibility of the rhesus monkey to measles appears to be subject to considerable variation, for it has not infrequently been found that when two animals were inoculated with the same material only one developed the disease, or that one gave a more marked reaction than the other.

As soon as we had definitely determined that the monkey was susceptible to measles by blood inoculation, we gave our attention to a study of the nature of the virus as it exists in the blood, and for this purpose made experiments to determine its filterability, resistance to drying, to heat, to freezing, and to age.

We found that the virus in measles blood is capable of passing through a Berkefeld filter, that it may resist desiccation for twenty-five and onehalf hours, that its infectivity was destroyed by heating at $55 \mathrm{C}$., for fifteen minutes, that it resisted freezing for twenty-five hours, and possibly retained some infectivity after keeping twenty-four hours at $15 \mathrm{C}$.

\section{NASAL AND BUCCAL SECRETIONS}

In order to test the infectivity of the nasal and buccal secretions from human cases of measles, we obtained such secretions from a patient with the disease and made serial inoculations with these secretions at different stages of the illness. We found that the secretions from this case were infective for the monkey at the time of the first appearance of the eruption, and again forty-eight hours later; that is, early in the fourth day and early in the sixth day of the disease, and that this infectivity was due to a living virus susceptible of transfer from monkey to monkey by blood inoculation.

In order to determine the duration of the infectivity of the secretions, we made inoculations in monkeys with the secretions collected at various periods of the disease from five additional patients.

Without going into the details of these experiments it is sufficient for the purposes of this paper to say that, viewing our experiments with secretions as a whole, it would appear that the nasal and buccal secretions in uncomplicated cases of measles may be at times, but are not always, infective for the monkey, and that the positive results were obtained only with secretions collected in the twenty-four- and forty-eight-hour period of the eruption.

Our experiments so far do not permit us to draw definite conclusions as to the duration of the infectivity of the secretions, but we believe that our results tend strongly to suggest a reduction, if not a total loss, of infectivity of the nasal and buccal secretions with the approach of convalescence. 


\section{INFECTIVITY OF THE "SCALE"}

We made three experiments" to determine the infectivity of the measles "scales" collected from human cases of the disease from four to seven days after the first appearance of the eruption. In not a single one of the monkeys - two in each experiment, or six altogether - was there the least evidence of a reaction. Alone, the negative results of these experiments, while highly suggestive, would hardly justify the conclusion that the desquamating epidermis is not infective for man; but, when viewed in the light of Mayr's negative results in children, we believe that the desquamating epithelium in measles does not, in itself, carry the vims of the disease.

\section{CULTURES}

We have made many cultures with measles blood known to be infective by monkey inoculations, but in no instance have we been able to obtain a growth that appears to have any etiologic relation to the disease. 